\title{
Pflege auf der Stroke Unit
}

\author{
1. Deutscher Schlaganfall-Kongress — Hohe Anforderungen an die Pflege, ohne \\ Konzept für diese komplexe Arbeit - das war lange die Situation an Stroke Units. \\ Inzwischen existiert eine spezifische, standardisierte und zertifizierte Fachweiterbildung \\ für Schwestern und Pfleger, entwickelt am Klinikum Minden.
}

\section{$\boldsymbol{\nabla}$} Als vor etwa zehn Jahren die ersten Stroke Units in Deutschland eröffnet wurden, begannen sie ihre Arbeit ohne Konzept für die Pflege akuter Schlaganfallpatienten. „Die Stationen waren im Aufbau sowie in Organisation, Diagnostik und Therapie primär ärztlich definiert“, sagte Dr. Jörg Glahn von der Neurologischen Klinik am Klinikum Minden. „Die Pflege musste sich in diese Situation integrieren. “Doch bereits in der Anfangsphase wurde deutlich: Inhalte der „normalen“ Pflegeausbildung reichten nicht aus, bestehende Pflegekonzepte waren eher auf lange Zeit ausgerichtet - etwa in der Rehabilitation und Sekundärversorgung in Pflegeheimen. Gerade aber die kurze Verweildauer des Schlaganfallpatienten mit akuter neurologischer Symptomatik und instabilen Vitalfunktionen auf einer Stroke-Unit stellt die Pflege vor komplexe verdichtete Aufgaben in extrem kurzer Zeit. Es bestand hoher Bedarf nach entsprechender Weiter- und Ausbildung.

In Minden begann eine Arbeitsgruppe aus Mitarbeitern der Stroke Unit, dem
Institut für Fort- und Weiterbildung und der ärztlichen Leitung die eigenen Erfahrungen zu bündeln und ein Fachweiterbildungskonzept zu entwickeln, das den gesamten Bereich von Ursache, Diagnostik bis Therapie umfasst. Damit werden auch die Mindestvoraussetzungen der Deutschen Schlaganfall-Gesellschaft für die Zertifizierung von Stroke Units erfüllt, die die systematische Weiterbildung aller Berufsgruppen fordert.

\section{Praxisnahe Weiterbildung}

Erfahrene Experten der täglichen Praxis vermitteln umfassende medizinische Grundlagen des Krankheitsbildes mit Kenntnissen in Neuroanatomie, Pathophysiologie, Diagnostik und Therapie. Spezielle pflegerische Inhalte reichen von der pflegerischen Überwachung bis zur ganzheitlichen rehabilitativen Pflege, mit Logopädie und Ergotherapie, Schluckdiagnostik und -therapie, Bobath, basale Stimulation oder Kinästhetik. Weiterhin wird die kommunikative Kompetenz für die tägliche Gesprächsführung im Team und mit Patienten und Angehörigen

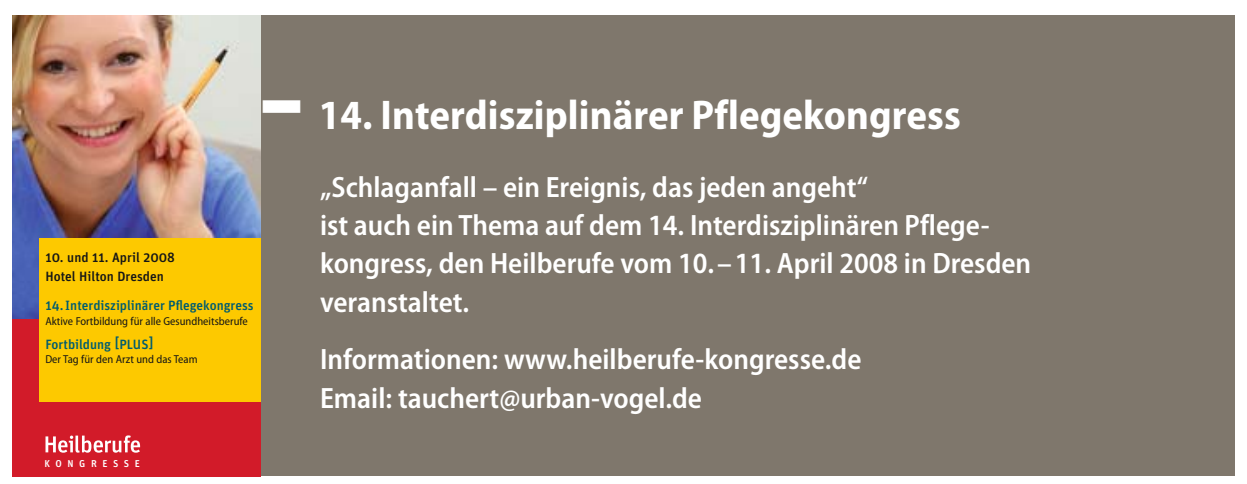

geschult. Die Teilnehmerzahl ist auf 25 begrenzt, um in einzelnen Gruppen auch praktisch optimal arbeiten zu können.

\section{Ausgebuchte Kurse}

Inzwischen wird der stark nachgefragte Kurs zwei Mal jährlich angeboten und geht über 240 Schulstunden in Blöcken von fünf beziehungsweise zehn Tagen. Interessierte, die noch nicht auf einer entsprechenden Station arbeiten, müssen zuvor auf einer zertifizierten Stroke Unit hospitieren. Den Abschluss bildet eine Prüfung im Rahmen eines Symposiums, für die jeder Teilnehmer ein spezielles Thema ausarbeitet, präsentiert und diskutiert. Angeboten wird der Kurs in fünf Zentren: außer in Minden noch in Hamburg, Berlin, Aue/Dresden und Karlsbad. Weitere Kliniken wollen das Konzept übernehmen. Weil viele Schwestern und Pfleger den Kurs aus eigener Tasche finanzieren müssen, appellierte Glahn abschließend an die Klinikchefs, die Finanzierung zu übernehmen. Denn die Teilnahme lohnt sich für alle Beteiligten: „Ärzte und Pflegekräfte arbeiten und kommunizieren auf dem selben Niveau. Das verbessert die Zusammenarbeit“, berichtete Glahn seine Erfahrungen. Auch wenn es besonders für junge Assistenzärzte häufig überraschend ist, wenn sie sehen müssen, dass die erfahrene Schwester zum Teil über detailliertere Kenntnisse verfügt als sie selbst.

- Michael Koczorek

1. Deutscher Schlaganfall-Kongress, Hannover, 9. und 10. November 2007 